\title{
Upward trend in the frequency of community-acquired methicillin-resistant Staphylococcus aureus as a cause of pediatric skin and soft tissue infections over five years: a cross-sectional study
}

\author{
Elif Böncüoğlu ${ }^{1 \oplus}$, Elif Kıymet ${ }^{1 \oplus}$, İlknur Çağlar ${ }^{1 \oplus}$, Yeliz Oruçç ${ }^{2 \oplus}$ \\ Nevbahar Demiray $^{2 \odot}$, Aybüke Akaslan Kara ${ }^{1 \oplus}$, Tülin Erdem ${ }^{3 \odot}$, \\ Gamze Gülfidan $^{3 \odot}$, İlker Devrim ${ }^{1 \odot}$, Nuri Bayram ${ }^{1 \odot}$ \\ ${ }^{1}$ Department of Pediatric Infectious Diseases, ${ }^{2}$ Infection Control Committeeand and ${ }^{3}$ Department of Microbiology, University of Health \\ Sciences Dr. Behçet Uz Children's Hospital, İzmir, Turkey.
}

\begin{abstract}
Background. The increasing prevalence of methicillin-resistant Staphylococcus aureus (MRSA) and its resistance to multiple antibiotics has become a serious challenge since the early 2000s. Especially, community-acquired MRSA (CA-MRSA) infections that appear mainly as skin and soft tissue infections (SSTIs) tend to increase worldwide. The objective of this cross-sectional study was to evaluate the trends in the frequency of SSTIs due to community-acquired S. aureus among children.
\end{abstract}

Methods. All children with SSTIs caused by culture positive community-acquired S. aureus during the period from 2013 to 2018 were included in this study. Data of the outpatients were collected from medical records. Annual alteration in frequencies of CA-MRSA and community-acquired methicillin-sensitive S. aureus (CAMSSA) were evaluated.

Results. A total of 112 cases was evaluated. Of these, 35 (31.25\%) were CA-MRSA. The rates of CA-MRSA had emerged from an increasing annual frequency of 9.5 cases per 10,000 SSTIs as of 2014 to 96.8 cases per 10,000 SSTIs in 2018. The ratio of cases with CA-MRSA to cases with CA-MSSA was $0-0.09$ in two years of the study period and increased to a maximum ratio of $0.6-0.72$ in the last two years. Consequently, the frequency of $S$. aureus in cases with SSTIs was significantly higher in 2016-2018 compared to the initial study period within the years of 2013-2015 [ $<<0.001$, relative risk increase: 7 (2.6-28.7) for CA-MRSA and $\mathrm{p}=0.002$, relative risk increase: 2.1 (1.2-3.5)]. Cases with CA-MRSA increased approximately eight-fold during the six-year-study period.

Conclusions. The rates of CA-MRSA in SSTIs among children increased significantly compared to CA-MSSA. The clinical impact of this increase should be evaluated, especially in patients with SSTI who are unresponsive to empirical treatment.

Key words: Methicillin-resistant Staphylococcus aureus, child, skin diseases, infectious, soft tissue infections.

Staphylococcus aureus is a common pathogen that causes most skin and soft tissue infections (SSTIs). Despite the initial description of methicillin-resistant Staphylococcus aureus

$\triangle \quad$ Nuri Bayram

nuribayram@gmail.com

Received 5th May 2020, revised 30th July 2020,

10th August 2020, accepted 13rd August 2020.
(MRSA) as a nosocomial pathogen in the $1960 \mathrm{~s},{ }^{1}$ it has become an outspread cause of communityassociated infections. Since the early 2000s, the increasing prevalence of MRSA and its resistance to multiple antibiotics has become a serious challenge. ${ }^{2}$ Besides invasive infections such as bacteremia, pneumonia, urinary tract infections; up to $96 \%$ of community-acquired MRSA (CA-MRSA) infections detected in children are SSTIs such as abscess, furuncle, 
and cellulitis. ${ }^{3-6}$ The prevalence of CA-MRSA infections has been reported with different characteristics in different populations worldwide. ${ }^{7,8}$ Recent studies showed the controversial rates of CA-MRSA infections, ranging from $70 \%$ in some regions of the United States to no cases in Finland. ${ }^{7,8}$ While the rates of healthcare-associated MRSA infections have recently declined due to infection control programs, CA-MRSA infections tend to increase worldwide. $^{9}$

In Turkey, the prevalence of SSTIs caused by CA-MRSA in children has been investigated in a limited number of studies and to date, there is no study focusing on a large-scale, long term alteration of the number of CA-MRSA infections in children. This cross-sectional study aimed to evaluate the changing frequencies of SSTIs caused by CA-MRSA among children in the study center, between the period of 2013-2018.

\section{Material and Methods}

\section{Study subjects}

This study includes outpatients with SSTI between January 1, 2013 and December 31, 2018 at Dr. Behçet Uz Children's Hospital a pediatric referral and tertiary care hospital in Izmir, Turkey, with an annual 600,000 outpatient visits and 24,000 hospitalizations in 2018 . The medical records of the patients were obtained from the hospital electronic information system. All children diagnosed with L02 and its subsections (L02.0, L02.1, L02.2, L02.3, L02.4, L02.8, and L02.9) according to the International Statistical Classification of Diseases 10th Revision (ICD10) were screened retrospectively from the medical records. Patients with SSTI in whom methicillin-sensitive $S$. aureus (MSSA) or MRSA were identified from wound swab or abscess cultures during outpatient clinic visits or hospital admissions. All culture positive patients compatible with the case definition mentioned below were enrolled in the final analysis of the study.

\section{Definitions}

Patients with community-acquired MRSA or MSSA infections were defined according to the criteria of the Centers for Disease Control and Prevention (CDC). ${ }^{10}$ Those were the cases, culture-confirmed within 72 hours of admission; having no history of previous hospitalization, surgery, dialysis, or residence in a long-term care facility, and having no permanent indwelling catheter present at the time of culture.

\section{Microbiological analysis}

Identification of the isolates was performed by standard microbiological procedures. Organisms were identified to the species level by catalase and coagulase test. Phoenix automated system (BD, Sparks, MD, USA) and VITEK-2 compact automated system (bioMérieux, Marcy l'Étoile, France) were used for MRSA identification and susceptibility testing. Detection of MRSA in both systems is based on both oxacillin and cefoxitin minimum inhibitory concentrations, interpreted according to The Clinical and Laboratory Standards Institute and European Committee on Antimicrobial Susceptibility Testing. ${ }^{11,12}$

\section{Statistical analysis:}

The data were analyzed using SPSS Statistics 17.0 (International Business Machines Corp, Armonk, NY) and MedCalc Version 11.6 (MedCalc Software BVBA, Ostend, Belgium) software. The Kolmogorov-Smirnov test was used to determine whether the distribution of continuous variables was approximately normal, and the Levene test was used to determine whether the assumption of homogeneity of variance was met. Baseline characteristics were given as medians and interquartile ranges (IQRs) for continuous variables, and numbers of cases and percentages were calculated for categorical variables. The Mann- Whitney U test was used to examine differences between groups in non-normally distributed continuous variables. Categorical variables were evaluated 
by the Fisher exact test for expected frequencies. Results were considered statistically significant when $\mathrm{p}<0.05$.

The number of CA-MRSA SSTI cases was calculated for each year. The rate of MRSA infections (with a 95\% Poisson confidence interval) for each year and the relative risk reduction (determined by comparing groups) were calculated and are given as percentages. The relative risk ratio was also calculated, and a $95 \%$ confidence interval was used for the incidence rate.

Ethical approval was obtained from the Local Ethical Committee of Behçet Uz Children's Hospital on July 18, 2019. (Report number: 2019/320) All experiments were carried out in compliance with relevant laws and guidelines by following the ethical standards of the Declaration of Helsinki.

\section{Results}

During the study period, a total of 4,234,165 patients were recorded in the outpatient clinics and the emergency department. Skin and soft tissue infections were diagnosed in 6,718 cases. The annual numbers of outpatients and SSTI cases are reviewed in Table I. Of these, S. aureus grew in 155 cultures. Thirty-three patients who did not meet the case definition criteria and 10 patients due to the inadequate data were excluded. As a result, 112 cases accepted as SSTI caused by community-acquired $S$. aureus were enrolled in the final evaluation.

Among the 112 isolates, 35 (31.25\%) were CA-MRSA, and 77 (68.75\%) were CA-MSSA. Clindamycin resistance was found in two of 35 $(5.7 \%)$ patients with SSTI due to CA-MRSA.

\section{The trend in frequencies of CA-MRSA and CA- MSSA}

In 2013, CA-MRSA was not detected in any of the SSTIs. However, CA-MRSA had emerged from an annual frequency of 9.5 cases per 10,000 SSTIs as of 2014 to 96.8 cases per 10,000 SSTIs in 2018. The frequencies of CA-MRSA and CAMSSA are shown in Table I. The frequencies of CA-MRSA and CA-MSSA infections were calculated with the Poisson 95\% confidence interval in each year and compared with the consecutive annual interval. No significant relative risk was present in these years $(p>0.05)$ meaning no increase compared with the previous year (data shown in Table II). However, the ratio of cases with CA-MRSA to cases with CA-MSSA was $0-0.09$ in two years of the study period and increased to a maximum ratio of $0.6-0.72$ in the last two years. Consequently, the frequency of CA-MRSA and CA-MSSA in cases with SSTIs was found to be significantly higher within the years of 2016-2018 compared to the initial study period $2013-2015(\mathrm{p}<0.001$

Table I. The annual rates of community-acquired Staphylococcus aureus in patients with skin and soft tissue infections.

\begin{tabular}{lllllll}
\hline Year & $\begin{array}{l}\text { Total number of } \\
\text { patients admitted } \\
\text { to the hospital }(\mathrm{n})\end{array}$ & $\begin{array}{l}\text { Number of } \\
\text { cases with } \\
\text { SSTIs }(\mathrm{n})\end{array}$ & $\begin{array}{l}\text { MRSA } \\
\text { positive } \\
\text { cases }(\mathrm{n})\end{array}$ & $\begin{array}{l}\text { MSSA } \\
\text { positive } \\
\text { cases }(\mathrm{n})\end{array}$ & $\begin{array}{l}\text { Prevalence of MRSA Prevalence of MSSA } \\
\text { per 10,000 cases } \\
\text { with SSTI }(\mathrm{n})\end{array}$ & $\begin{array}{l}\text { per 10,000 cases } \\
\text { with SSTI (n) }\end{array}$ \\
\hline 2013 & 582,067 & 539 & 0 & 5 & 0 & 92.7 \\
2014 & 636,396 & 1048 & 1 & 11 & 9.5 & 104.9 \\
2015 & 657,147 & 1153 & 3 & 8 & 26 & 69.3 \\
2016 & 752,123 & 1112 & 6 & 15 & 53.9 & 134.8 \\
2017 & 788,702 & 1239 & 12 & 20 & 96.8 & 161.4 \\
2018 & 817,730 & 1627 & 13 & 18 & 79.9 & 110.0 \\
\hline Total & $4,234,165$ & 6718 & 35 & 77 & 52.1 & 114.6 \\
\hline
\end{tabular}

SSTI: skin and soft tissue infections, MRSA: methicillin-resistant Staphylococcus aureus, MSSA: methicillin-sensitive Staphylococcus aureus. 
Table II. Comparison of poisson 95\% confidence interval of cases with community-acquired Methicillinresistant Staphylococcus aureus and Methicillin-sensitive Staphylococcus aureus by consecutive years.

\begin{tabular}{lll}
\hline \multirow{2}{*}{ Years } & \multicolumn{2}{l}{ Comparison throughout consecutive years } \\
\cline { 2 - 3 } & CA-MRSA & CA-MSSA \\
\hline $2013-2014$ & $\mathrm{p}=0.4744(95 \%$ CI: $0-75.82)$ & $\mathrm{p}=0.8(95 \%$ CI: $0.2-2.7)$ \\
$2014-2015$ & $\mathrm{p}=0.3651(95 \%$ CI: $0.006-4.5)$ & $\mathrm{p}=0.36(95 \%$ CI: $0.5-4.3)$ \\
$2015-2016$ & $\mathrm{p}=0.2917(95 \%$ CI: $0.44-12.8)$ & $\mathrm{p}=0.12(95 \%$ CI: $0.7-5.2)$ \\
$2016-2017$ & $\mathrm{p}=0.2351(95 \%$ CI: $1.7-15.9)$ & $\mathrm{p}=0.5(95 \%$ CI: $0.3-1.7)$ \\
$2017-2018$ & $\mathrm{p}=0.63(95 \%$ CI: $0.34-1.9)$ & $\mathrm{p}=0.2421(95 \%$ CI: $0.34-1.36)$ \\
\hline
\end{tabular}

CA-MRSA: community-acquired methicillin-resistant Staphylococcus aureus, CA-MSSA: community-acquired methicillinsensitive Staphylococcus aureus.

and $\mathrm{p}=0.002$ consecutively.) Between the two periods, a 5-fold increase in the frequency of CA-MRSA and a 1.5-fold increase in the frequency of CA-MSSA were observed. During the six-year period, while the frequencies of the cases with SSTIs associated with CA-MSSA fluctuated, cases with CA-MRSA increased approximately eight-fold.

\section{Discussion}

Although infections caused by CA-MRSA is an emerging public health problem worldwide, a limited number of studies have been published on CA-MRSA prevalence in SSTIs in children. ${ }^{13,14}$ This study is one of the largest series of children with SSTIs. It is also the first to investigate the changing frequency of CA-MRSA in the etiologic spectrum of pediatric SSTIs in Turkey. It clearly points out that the rate of CA-MRSA in SSTIs has steadily increased in less than a decade.

In the last few decades, CA-MRSA infections have increased worldwide. In a study from the United States, the incidence of CA-MRSA associated SSTIs in adult patients was reported to increase from 24.0 cases per 100,000 people to 164.2 cases per 100,000 people over a fiveyear study period..$^{13}$ In another study, similarly, the annual prevalence of adult patients with CA-MRSA was reported to have significantly increased from $8.9 \%$ in 1996 to $39.6 \%$ of MRSA cultures in 2005. ${ }^{14}$ A study from Saudi Arabia which also included pediatric cases indicated that the prevalence of CA-MRSA infections increased from 9.9 to 67 per 10,000 admissions from 2001 to 2008, and the percentage of CAMRSA in MRSA isolates increased from $20 \%$ to $59 \% .{ }^{15}$ As in adult studies, a study concerning children indicated that the rates of CAMRSA infections increased from 12.2/10,000 to $145 / 10,000$ during a 10 -year period. ${ }^{16}$ Compatible with the literature, the data of the current study also revealed that CA-MRSA rates in SSTIs had increased from 9.5 to 79.9 per 10,000 cases between 2014 and 2018, suggesting a trend similar to those worldwide.

In a 2017 report by World Health Organization (WHO), the consumption of antibiotics in Turkey was among the highest across the WHO European region. ${ }^{17}$ The increase in the rates of CA-MRSA in SSTIs might be a reflection of the increased use of antibacterials. This resistance may pose a risk of treatment failure since most of the community-acquired SSTIs in children outpatients are treated with antimicrobial drugs such as sulbactam-ampicillin or amoxicillinclavulanate which the community-acquired S. aureus strains tend to be resistant to. ${ }^{18,19}$ Considering the high frequency of MRSA in the current study, it is thought that commonly used antimicrobials in our daily practice may not be effective in almost one-third of CA-MRSArelated SSTIs, suggesting that the selection of alternative antimicrobial drugs, such as clindamycin or rifampin is necessary for the initial treatment in anticipation of CA-MRSA. Only two of the 35 CA-MRSA cases in the study were resistant to clindamycin, suggesting 
that clindamycin can be a favorable choice for SSTIs despite its side-effects and reported clinical failure due to inducible clindamycin resistance. ${ }^{20}$

Several considerations should be noted when interpreting the results. First, this was a retrospective study, which has inherent limitations when compared to randomized clinical trials. Second, the patients with SSTI were selected based on the current ICD codes, so the total SSTI numbers may be slightly different due to incorrectly entered diagnostic codes. Third, the study results reflect a single-center experience. Multicenter studies may yield more reliable data to support the increasing trend of resistance in community-acquired $S$. aureus infections.

In conclusion, this study showed that CA-MRSA frequency in the etiology of SSTI in children has increased compared to that of CA-MSSA. The clinical impact of this increase should be taken into account especially in the patients with SSTI, who are unresponsive to empirical treatment.

\section{REFERENCES}

1. Shanson DC. Staphylococcal infections in hospital. Br J Hosp Med 1986; 35: 312-320.

2. Chambers HF. The changing epidemiology of Staphylococcus aureus. Emerg Infect Dis 2001; 7: 178-182.

3. Purcell K, Fergie J. Epidemic of community-acquired methicillin-resistant Staphylococcus aureus infections: a 14-year study at Driscoll Children's Hospital. Arch Pediatr Adolesc Med 2005; 159: 980985.

4. Fortunov RM, Hulten KG, Hammerman WA, Mason EO Jr, Kaplan SL. Community-acquired Staphylococcus aureus infections in term and nearterm previously healthy neonates. Pediatrics 2006; 118: 874-881.

5. Kaplan SL, Hulten KG, Gonzalez BE, et al. Three-year surveillance of community-acquired Staphylococcus aureus infections in children. Clin Infect Dis 2005; 40: 1785-1791.
6. Fortunov RM, Hulten KG, Hammerman WA, Mason EO Jr, Kaplan SL. Evaluation and treatment of community-acquired Staphylococcus aureus infections in term and late-preterm previously healthy neonates. Pediatrics 2007; 120: 937-945.

7. Grundmann H, Aires-de-Sousa M, Boyce J, Tiemersma E. Emergence and resurgence of meticillin-resistant Staphylococcus aureus as a public-health threat. Lancet 2006; 368: 874- 885.

8. Peltola $H$, Pääkkönen $M$, Kallio $P$, Kallio MJ; Osteomyelitis-Septic Arthritis (OM-SA) Study Group. Prospective, randomized trials of 10 days versus 30 days of antimicrobial treatment, including a short-term course of parenteral therapy, for childhood septic arthritis. Clin Infect Dis 2009; 48: 1201-1210.

9. David MZ, Daum RS. Community-associated methicillin-resistant Staphylococcus aureus: epidemiology and clinical consequences of an emerging epidemic. Clin Microbiol Rev 2010; 23: 616-687.

10. Maree C, Daum RS, Boyle-Vavra S, Matayoshi K, Miller LG. Community-associated methicillinresistant Staphylococcus aureus isolates and healthcare-associated infections. Emerg Infect Dis 2007; 13: 236-242.

11. Clinical and Laboratory Standards Institute (CLSI). Performance Standards for Antimicrobial Susceptibility Testing. (28th ed) CLSI Supplement M100 (ISBN 1-56238-838-X [Print]; ISBN 1-56238839-8 [Electronic]). Pennsylvania: Clinical and Laboratory Standards Institute, 2018. Available at: http://file.qums.ac.ir/repository/mmrc/CLSI-2018M100-S28.pdf (Accessed on April 14, 2020).

12. EUCAST guidelines for detection of resistance mechanisms and specific resistances of clinical and/or epidemiological importance. Available at: https://pdfs.semanticscholar. org/8fa9/0d4e48d77c9fb0e33a4e700d45cdde09b232. pdf (Accessed on April 14, 2020).

13. Hota B, Ellenbogen C, Hayden MK, Aroutcheva A, Rice TW, Weinstein RA. Community-associated methicillin-resistant Staphylococcus aureus skin and soft tissue infections at a public hospital: do public housing and incarceration amplify transmission? Arch Intern Med 2007; 167: 1026-1033.

14. McMullen KM, Warren DK, Woeltje KF. The changing susceptibilities of methicillin-resistant Staphylococcus aureus at a Midwestern hospital: the emergence of "community-associated" MRSA. Am J Infect Control 2009; 37: 454-457.

15. Bukharie HA. Increasing threat of communityacquired methicillin-resistant Stapylococcus aureus. Am J Med Sci 2010; 340: 378-381. 
16. Ensinck G, Ernst A, Lazarte G, et al. Communityacquired methicillin-resistant Staphylococcus aureus infections: 10-years' experience in a children's hospital in the city of Rosario, Argentina. Arch Argent Pediatr 2018; 116: 119-125.

17. World Health Organization. Central Asian and Eastern European Surveillance of Antimicrobial Resistance. Annual report 2017. (Available at: https://www.euro.who.int/_data/assets/ pdf_file/0005/354434/WHO_CAESAR_ AnnualReport_2017.pdf) (Accessed on April 14, 2020).

18. Montravers P, Snauwaert A, Welsch C. Current guidelines and recommendations for the management of skin and soft tissue infections. Curr Opin Infect Dis 2016; 29: 131-138.
19. Stevens DL, Bisno AL, Chambers HF, et al. Practice guidelines for the diagnosis and management of skin and soft tissue infections: 2014 update by the infectious diseases society of America. Clin Infect Dis 2014; 59: 147-159.

20. Frank AL, Marcinak JF, Mangat PD, et al. Clindamycin treatment of methicillin-resistant Staphylococcus aureus infections in children. Pediatr Infect Dis J 2002; 21: 530-534. 